\title{
Assessment of Mechanical Cardiac Function in Elite Athletes
}

\author{
J. Patrick Neary ${ }^{*}$, David S. MacQuarrie ${ }^{1}$, Veronica Jamnik ${ }^{2}$, Norman Gledhill ${ }^{2}$, Scott Gledhill ${ }^{3}$ and \\ Edward F.G. Busse ${ }^{1}$
}

${ }^{1}$ University of Regina, Regina, SK, Canada

${ }^{2}$ York University, Toronto, ON, Canada

${ }^{3}$ Toronto General Hospital, Toronto, ON, Canada

\begin{abstract}
Hypertrophic cardiomyopathy (HCM) is the number one cause of sudden cardiac death in elite athletes. This project used resting 12-lead electrocardiography (ECG) and ballistocardiography (BCG) to assess cardiac cycle timing events as simple screening techniques to rule out cardiac abnormalities for the safety of a group of elite ice hockey players. Clinical cardiac (ECG) and physiological (maximal aerobic power $\left[\mathrm{VO}_{2} \mathrm{max}\right.$ ], anaerobic [Wingate peak power, Watts] and musculoskeletal strength) data is presented here on an elite group of ice hockey players ( $\mathrm{n}=34 ;$ age $=17-18 \mathrm{yrs}$ ) that participated in a professional medical and fitness evaluation. Subsequently one subject was diagnosed with \#1 Apical $\mathrm{HCM}$ and his cardiac data is compared with the group. The HCM subject performed all fitness testing and was determined to be physically fit $\left(\% \mathrm{BF}=7.2 \% ; \mathrm{VO}_{2} \mathrm{max}=59.4 \mathrm{~mL} \cdot \mathrm{kg}^{-1} \cdot \mathrm{min}^{-1}\right.$; Wingate peak power output $=15.1 \mathrm{Watt} \cdot \mathrm{kg}^{-1}$; Heart Rate $\max =200$ beats $\bullet \mathrm{min}^{-1}$ ). However, the ECG showed extreme voltage and deeply inverted T-waves, and the BCG showed abnormal waveform complexes and cardiac timing events in comparison to the group means. Mean BCG systolic timing events for isovolumic contraction time $(54.7 \pm 7.1$ vs $49.5 \pm 12.4 \mathrm{msec})$, acceleration time $(49.1 \pm 1.8 v s 56.3 \pm 9.1 \mathrm{msec})$, diastole $(470.8 \pm 25.3$ vs $531.4 \pm 166.7 \mathrm{msec})$, and isovolumic relaxation time $(88.5 \pm 7.4$ vs $100.8 \pm 16$ msec $)$ were significantly different $(\mathrm{p}<0.05)$. Atrial systole amplitude was statistically higher for this subject $(9.2 \pm 3.7 \mathrm{vs} 5.3 \pm 3 \mathrm{mG})$. Subsequent follow-up assessment showed abnormal echocardiogram (Echo) dimensions (ventricular septum [12mm]; posterior wall [16 mm]), velocities (mitral valve deceleration [233 msec], LV systolic strain [14\%]), and volumes (LV stroke volume $\left[38 \mathrm{~mL} \cdot \mathrm{metre}^{-1}\right.$ body surface area]), with normal E:A ratio (1.75) and LV ejection fraction (62\%). Cardiac magnetic resonance imaging (MRI) showed apical septal wall thickness (24-25 mm) in the HCM player. In conclusion, BCG was able to corroborate a cardiac abnormality that was later confirmed with echocardiography and MRI, suggesting that BCG is a potential technology to detect anomalies that alter cardiac timing and amplitude.
\end{abstract}

Keywords: Hypertrophic cardiomyopathy, elite athlete, ballistocardiography, echocardiography, cardiac MRI.

\section{INTRODUCTION}

Chronic intensive or extreme endurance training is associated with very distinct structural and functional cardiac adaptations, some of which may lead to variations of hypertrophic cardiomyopathy (HCM). The complexity of diagnosis of HCM is clinically problematic [1,2]. One form of HCM termed the "athletic heart" [3] is characterized by both cardiac tissue and cardiovascular system adaptations which allow intense exercise to be sustained for prolonged periods of time, such as a marathon or triathlon, or for intermittent sports such as basketball or ice hockey. Sports such as ice hockey for example require high levels of all fitness parameters including aerobic, anaerobic and muscular strength and therefore extreme levels of exercise training are required that this could lead to an "athletic heart". Another form of HCM, a pathological form, is the result of a genetic disorder that is prevalent in approximately $0.2 \%$ of the population $[4,5]$. It is characterized by electrically unstable myocardial substrate, a disorganized cellular architecture, ischemia, cell death, and replacement scarring $[2,6]$.

*Address correspondence to this author at the Faculty of Kinesiology \& Health Studies, University of Regina, Regina, SK, S4S0A2, Canada; Tel: 306-585-4844; Fax: 306-585-4854; E-mail: patrick.neary@uregina.ca
Although the prevalence rate of pathological $\mathrm{HCM}$ is extremely low, the literature suggests that it accounts for 36$50 \%$ of "sudden unexpected cardiac death" in competitive athletes from all sports [7, 8]. However, recent reports suggest that sudden cardiac death in athletes is extremely low ( $\sim 1$ in 400,000 athletes) [2] and is more prevalent in males than females [9]. Previous research conducted in regions of Italy that are well known for congenital cardiac complications have screened young athletes since the late 1970 's to further reduce the rate of mortality [10]. However, there is much controversy in the literature as to whether preparticipation screening should be conducted on young athletes because of the low incidence rate of HCM, and the cost effectiveness of performing comprehensive testing [7, 11-16].

There are a number of non-invasive techniques used to assess athletes and diagnose HCM, including electrocardiography (ECG), echocardiography (Echo), and cardiac magnetic resonance imaging (MRI). Pathological HCM is generally asymptomatic at rest, and is characterized by a number of cardiac abnormalities. The more common ECG abnormalities are ST-T changes, including prominent T-wave inversion, abnormal $Q$ waves, and abnormal progression of the $\mathrm{R}$-wave voltages across the precordial 
leads, and at times, signs of left atrial enlargement [8, 17, 18]. However, the ECG is not always reliable to assess pathological HCM [19], and thus cardiac problems can potentially go undetected. Standard two-dimensional guided M-mode and Doppler Echo are commonly used to evaluate left ventricular morphology and function [20]. With Echo, pathological HCM is characterized by left and right chamber alterations including LV septal anomaly, posterior wall thickness, impaired ventricular diastolic filing, increased ventricular stiffness and mitral valve systolic anterior motion (SAM). Cardiac MRI is likewise useful to localize and quantify cardiac hypertrophy, as it is capable of measuring both ventricles to detect biventricular hypertrophy [21-23]. All of these methods have their distinct advantages, and when combined, provide the clinician with a thorough and reliable methodology for diagnosing HCM [24]. However, some of these techniques are not readily accessible and are time consuming, especially if many individuals are being assessed at the same time, such as for Olympic, national or professional sports teams.

Ballistocardiography or seismocardiography was used in the 1940's, 50's and 60's to record the ultra low frequency vibrations of the heart, thus allowing the measurement of the cardiac cycle events including timing, durations and amplitudes of contraction [25-28]. Later, the technology was refined by Salerno and colleagues to show that cardiac cycle timing events at rest could be recorded reliably using this technology [29, 30]. Today, because of micro-processors and advanced computer technology a digitized signal representing the cardiac cycle is possible. Hence, the BCG is a recording of the motion of the lower sternum resulting from the movement and contraction of the heart. The primary data sensed by the BCG device are the directional units of acceleration and time which can be interpreted in relation to the classic ECG cardiac time intervals. Thus, the BCG illustrates the mechanical performance (i.e., timing and force) of contraction and relaxation of the heart during each cardiac cycle. However, because an abnormal 12-lead ECG is not always found in HCM patients [19, 31, 32], and ECG is not always reliable in providing clinical evidence for other cardiac abnormalities that are potentially lethal [33], we used BCG to evaluate 34 elite ice hockey players to examine its potential utility as a screening and clinical research device. Ballistocardiography has a distinct advantage over ECG as it can provide additional information about the cardiac cycle timing events, durations and contractility changes, and it has been correlated with Echo [34]. Furthermore and perhaps most importantly, only resting BCG samples are needed to evaluate potential cardiac complications and the assessment can be completed in a matter of minutes.

Given the fact that elite athletes such as hockey players are potentially at risk for HCM, and given the potential of not detecting HCM on routine medical screening [2, 33] without the aid of Echo [11, 24, 35], we hypothesized that if our group of elite athletes had cardiac complications then BCG could detect cardiac changes under resting conditions. Thus, the primary purpose of this study was to screen a group of elite ice hockey players using BCG and ECG to detect potential cardiac problems, and a secondary purpose was to gather normative cardiac data using BCG on elite ice hockey players for subsequent comparison with other athletic groups. To our knowledge, BCG has not been used for screening of elite athletes. It was fortuitous during our medical screening that we did detect a cardiac abnormality, and we present these data here.

\section{METHODS}

\section{Subjects}

In this study, written informed consent was obtained from 34 elite male ice hockey players (age $=18.0 \pm 0.5 \mathrm{yrs}$; $\mathrm{Ht}=186.9 \pm 5.4 \mathrm{~cm}$; Mass $=86.8 \pm 6.9 \mathrm{~kg}$ ) who were invited to undergo a comprehensive medical and fitness screening protocol. The physical characteristics of the group were not different from the single subject that was subsequently diagnosed with HCM. The nature and objectives of this study precluded the need for a control group.

\section{Experimental Protocol}

\section{Medical Examination}

Following the signed informed consent, a medical examination was performed by the attending physician who included a resting 12-lead ECG (ELI 100/ST Mortara Instrument Inc; Milwaukee, WI, USA) and auscultation of the pericardium. In addition, a technician conducted a $30-\mathrm{sec}$ BCG using a ballistocardiograph device (Heart Force Medical Inc., Vancouver, BC, Canada). At the time of the examination, the attending physician who conducted the auscultation and reviewed the ECG suspected an abnormality in one of the hockey players. The BCG technician also detected the anomaly. The subject was allowed to continue all fitness testing (see below) but under the watchful eye of trained certified specialists with an emergency physician and defibrillator present at all times. After the conclusion of all testing and medical monitoring, further evaluation of the 12-lead ECG (Fig. 1) by a cardiologist was performed and the subject was then referred for extensive cardiac analysis by a cardiac consultant (Echo, cardiac MRI, haematology, stress exercise test) as standard medical procedure. The comprehensive cardiac follow-up assessment was performed within 3 weeks of the initial medical examination. No other subjects presented with abnormal cardiac characteristics.

\section{HCM Subject Medical History}

The subject confirmed that he was normally healthy, and asymptomatic, had not reported any chest pressure, chest pain, dyspnea, palpitations, syncope, or near syncope previously during any physical exertion. There was no known family history of pathological HCM at the time of assessment, but hypertension and hyperlipidemia are reported in the family. The subject reported not using tobacco, alcohol, or other drugs. He had a normal lipid and haematology profile. However, the subject had abnormally high B-Type natriuretic peptide (BNP) (96 pg. $\mathrm{mL}^{-1}$; normal $\left.=<35 \mathrm{pg} \cdot \mathrm{mL}^{-1}\right)$. No prior cardiac complications had been reported previously.

\section{Ballistocardiography (BCG)}

A single 30 -sec BCG was recorded simultaneously with 12-lead ECG under quiet resting conditions in the supine position during the medical examination which was performed prior to any physical exercise. The BCG sensor was placed $1 \mathrm{~cm}$ above the xiphoid process on the sternum 


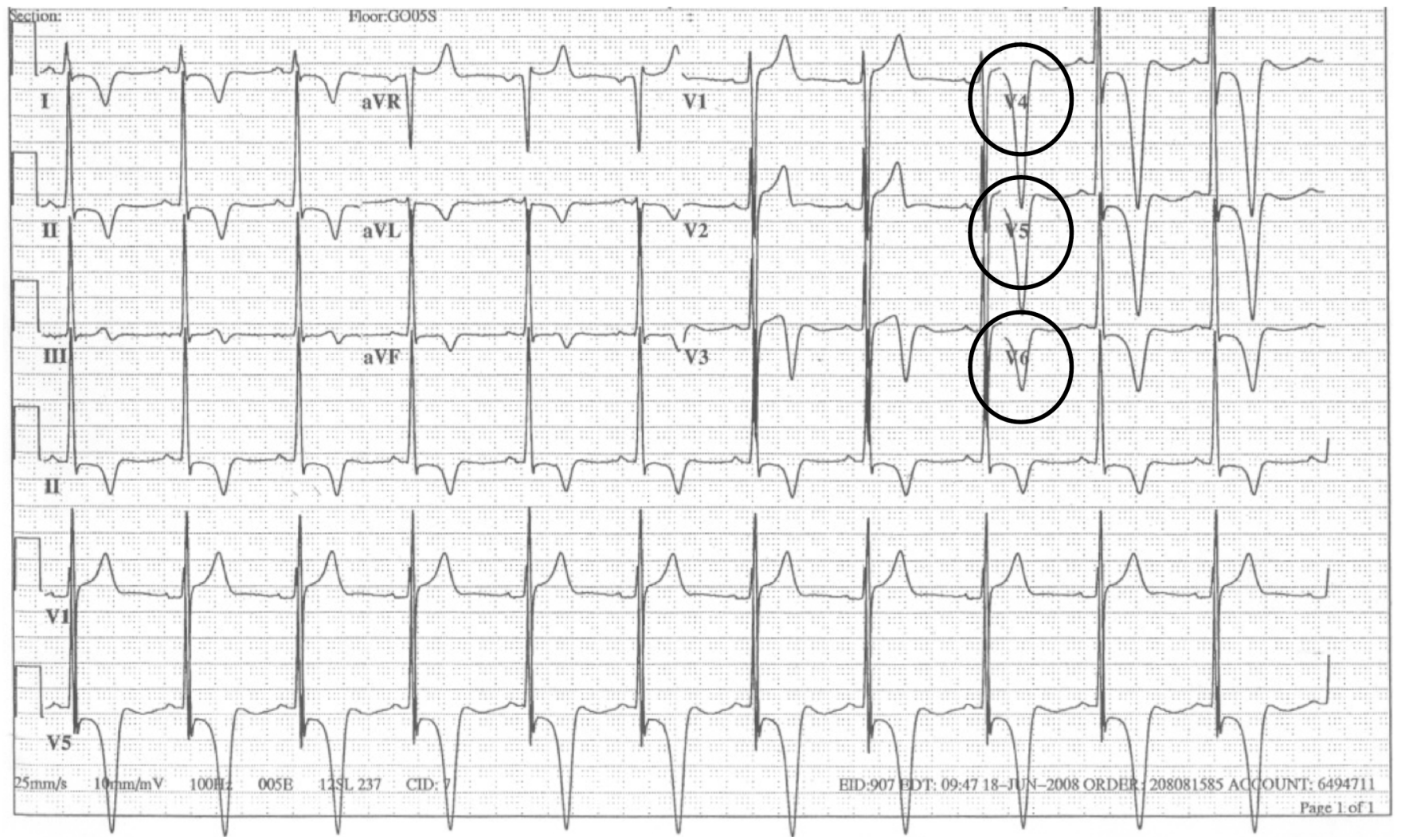

Fig. (1). 12-lead electrocardiogram (ECG) of the HCM subject showing normal sinus rhythm. However, note ST segment abnormality and inverted T-wave in Leads I, II, III, V4, V5 and V6.

of the chest. All subjects were asked to "completely relax and breathe normally" during the recording. The BCG system comprises a 20-gram factory calibrated sensor (to record the mechanical functioning of the heart), a recording device (with Bluetooth ${ }^{\mathrm{TM}}$ technology for telemetry data transfer), and a laptop computer containing the proprietary software for storage and subsequent analysis of the BCG waveforms. The nomenclature described by Scarborough et al., [36] and Crow et al., [34] was used to analyse the BCG waveform and is described in the caption for Fig. (2).

The BCG device provides a record of the motion of the lower sternum resulting from the movement of the heart during each cardiac cycle. The movement is sensed by an accelerometer and processed digitally to be displayed with the accelerator amplitudes as the vertical axis and time as the horizontal axis. Measurement of acceleration is expressed in milligravity $(\mathrm{mG})$ units and time is recorded in milliseconds (msec). A Lead-II ECG configuration is sensed and recorded simultaneously with the BCG within the sensor device.

The ballistocardiogram was later analysed off-line for cardiac cycle systolic and diastolic timing events (Q-wave of ECG to mitral valve close, $Q$ to aortic valve open, $Q$ to Jwave (rapid ejection period), Q to mitral valve open, $\mathrm{Q}$ to early diastolic filling wave, Q to late diastolic filling wave, systole, diastole, isovolumic contraction time [IVCT], pressure $1 / 2$ time $\left[\mathrm{P}^{1} / 2 \mathrm{~T}\right]$, isovolumic relaxation time [IVRT]), E:A ratio and amplitude (G-wave, AVO, MVO, ED-wave, LD-wave) by the BCG technician who was "blinded" to the Echo and MRI data. On average, $31 \pm 6$ waveforms were annotated then averaged $( \pm$ SD) for each cardiac variable list above. In our laboratory, intra-observer and inter-observer variability is $\mathrm{r}=0.96$ and 0.94 , respectively. See Fig. (2) for the nomenclature listed here [34].

\section{Fitness Testing}

Standardized fitness testing protocols performed according to Burr et al., [37] were used to assess all fitness parameters used in this study. A 30 -sec modified Wingate test performed on the Monark 834E Wingate ergometer was used to assess anaerobic performance, while aerobic power $\left(\mathrm{VO}_{2} \max \right)$ was assessed on the Monark 828 cycle ergometer (Table 1a). The assessment of musculoskeletal fitness included grip strength, a $150-1 \mathrm{~b}$ bench press in time to a metronome (set at $50 \mathrm{~b} \cdot \mathrm{min}^{-1}$ ), pull and push strength (Table 1b), vertical jump and standing long jump, push-ups, curlups, and trunk flexibility (sit \& reach) (Table 1c). Telemetry heart rate (Suunto, Ogden, UT, USA) was recorded continuously throughout the fitness testing.

A stress-ECG test was also performed on the HCM subject during follow-up analysis with the metabolic pulmonary gas exchange results confirming those attained at the combine medical and fitness testing. The physiological fitness testing data included here confirmed that the HCM subject was physically fit in comparison to his cohort.

\section{Echocardiography (Echo)}

After the initial examination, a comprehensive twodimensional apical visualization, M-mode and Doppler echocardiography was performed to assess ventricular morphology, dynamic left ventricular outflow obstruction, and systolic anterior motion of the mitral apparatus on only 

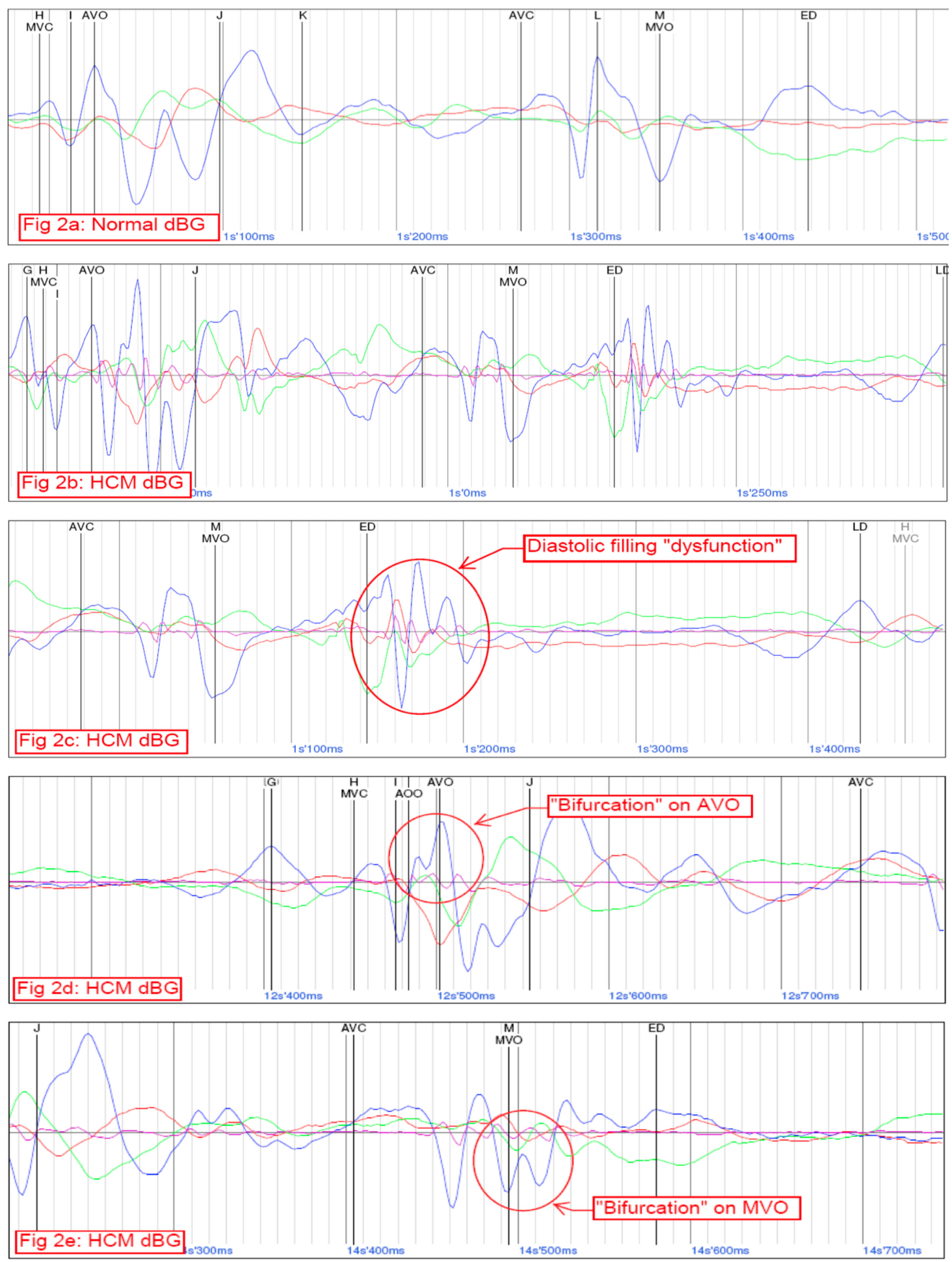

Fig. (2). Ballistocardiogram (BCG) illustrating the cardiac cycle timing events and waveform amplitudes. (a) A representative ("normal") BCG from one hockey player of the cohort group; (b) BCG from the hypertrophic cardiomyopathy (HCM) hockey player showing the complete cardiac cycle; (c) BCG from the HCM hockey player showing the aberrant waveform immediately following the early diastolic filling (ED) waveform; (d) BCG from the HCM hockey player showing the bifurcation on the aortic valve open (AVO) waveform; (e) BCG from the HCM hockey player showing the bifurcation on the mitral valve open (MVO) waveform.

the HCM player using commercially available ultrasound equipment. All images were acquired with the subject lying in the left lateral decubitas position. The heart chambers volume, flow velocity, dimensions, and valve functions are presented in Table $\mathbf{2}$ and Fig. (5) (see Results). The same certified Echo sonographer performed all measurements under the direction of the attending cardiac consultant with procedures reported previously [38, 39], and both were "blinded" to the BCG data. 
Table 1a. Anaerobic and Aerobic Fitness Variable Results for the Hypertrophic Cardiomyopathy (HCM) Subject and Group Results for the 33 Elite Male Ice Hockey Players (Values are Mean \pm SD)

\begin{tabular}{|c|c|c|c|}
\hline & Measured Variable & HCM Player & Group Data \\
\hline \multirow{5}{*}{ Anaerobic Fitness } & Peak Power Output (Watts) & 1283.8 & $1146.8(118)$ \\
\hline & Peak Power Output $\left(\mathrm{W} \cdot \mathrm{kg}^{-1}\right)$ & 15.1 & $13.3(1.0)$ \\
\hline & Mean Power Output (Watts) & 977.5 & $879.1(85.3)$ \\
\hline & Mean Power Output $\left(\mathrm{W} \cdot \mathrm{kg}^{-1}\right)$ & 11.5 & $10.2(0.6)$ \\
\hline & Fatigue Index \% Drop-Off From Peak & 52.5 & $50.5(5.4)$ \\
\hline \multirow[b]{3}{*}{ Aerobic Fitness } & $\mathrm{VO}_{2} \max \left(\mathrm{L} \cdot \min ^{-1}\right)$ & 5.05 & $4.79(0.5)$ \\
\hline & $\mathrm{VO}_{2} \max \left(\mathrm{mL} \cdot \mathrm{kg}^{-1} \cdot \mathrm{min}^{-1}\right)$ & 59.4 & $55.2(4.5)$ \\
\hline & Peak HR $\left(b \cdot \min ^{-1}\right)$ & 200 & $190(7)$ \\
\hline
\end{tabular}

Table 1b. Lower Body Musculoskeletal Fitness Results for the Hypertrophic Cardiomyopathy (HCM) Player and Group Results for the 33 Elite Male Ice Hockey Players (Values are Mean \pm SD)

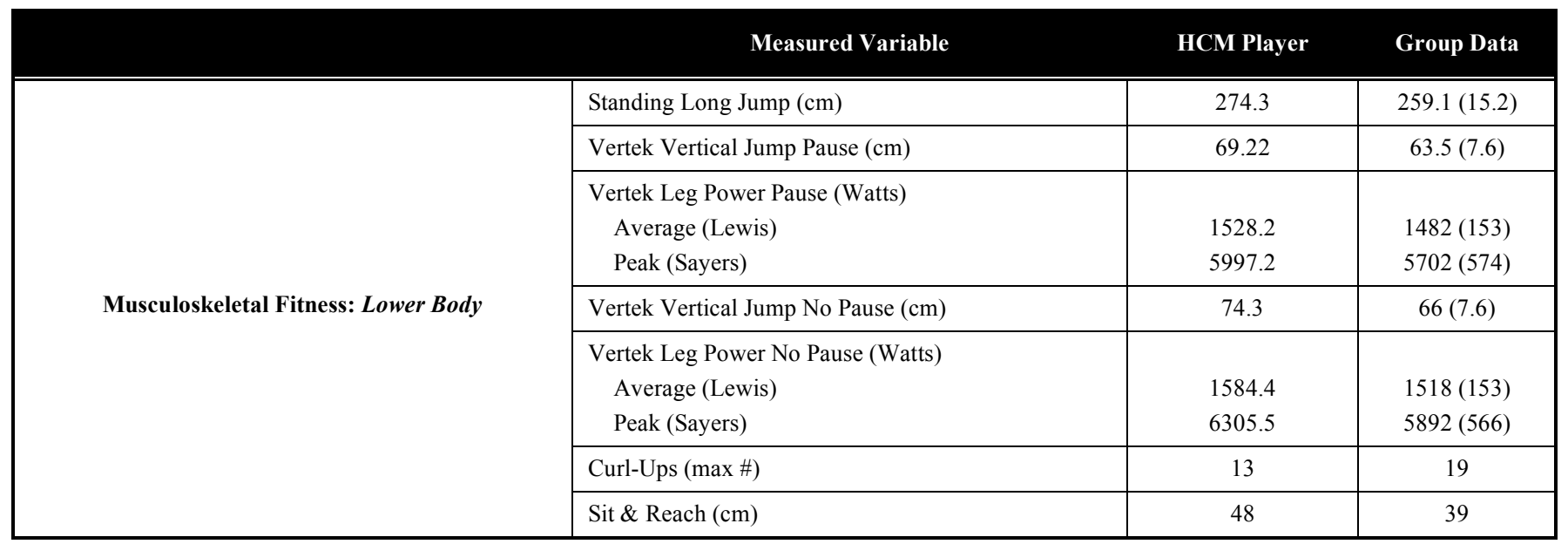

Table 1c. Upper Body Musculoskeletal Fitness Results for the Hypertrophic Cardiomyopathy (HCM) Player and Group Results for the 33 Elite Male Ice Hockey Players (Values are Mean \pm SD)

\begin{tabular}{|c|c|c|c|}
\hline \multirow{8}{*}{ Musculoskeletal Fitness: Upper Body } & Right Hand Grip (lb) & 154 & $125(14)$ \\
\hline & Bench Press (\# of 150-lb reps) & 12 & $11(5)$ \\
\hline & Bench Press $\left(\mathrm{lb} \cdot \mathrm{lb}^{-1}\right.$ body mass $)$ & 9.63 & $8.70(3)$ \\
\hline & Push-Ups × Body Weight (lb) & 5984 & $5264(1494)$ \\
\hline & Push Strength (lb) & 220.1 & $258(48)$ \\
\hline & Push Strength (lb $\cdot 1 b^{-1}$ body mass) & 1.18 & $1.36(0.26)$ \\
\hline & Pull Strength (lb) & 275.6 & $258(27)$ \\
\hline & Pull Strength (lb $\cdot 1 b^{-1}$ body mass) & 1.47 & $1.36(0.15)$ \\
\hline
\end{tabular}


Table 2. Echocardiography and Cardiac MRI Results for the Hypertrophic Cardiomyopathy (HCM) Subject in Comparison to Normal Ranges. Asterisks Denotes Different from the Normal Range

\begin{tabular}{|c|c|c|c|}
\hline & Measured Variable & HCM Subject & Normal Range \\
\hline \multirow{9}{*}{ Left Ventricular Function } & LV End Diastolic Volume $\left(\mathrm{mL} \cdot \mathrm{m}^{2}\right)$ & 55 & $56-108$ \\
\hline & LV End Systolic Volume $\left(\mathrm{mL} \cdot \mathrm{m}^{2}\right)$ & 17 & $9-41$ \\
\hline & LV Stroke Volume $\left(\mathrm{mL} \cdot \mathrm{m}^{2}\right)$ & $38 *$ & $40-72$ \\
\hline & LV Ejection Fraction (\%) & 68 & $57-81$ \\
\hline & Dimensions (diastole) (mm) & 51 & $42-56$ \\
\hline & Dimensions (systole) $(\mathrm{mm})$ & 33 & $25-37$ \\
\hline & LV Mass (g) & 300 & \\
\hline & LV End Diastolic Mass $\left(\mathrm{g} \cdot \mathrm{m}^{2}\right)$ & $128^{*}$ & $45-81$ \\
\hline & LV Mass Index $\left(\mathrm{g} \cdot \mathrm{m}^{2}\right)$ & 143 & $50-70$ \\
\hline \multirow{4}{*}{ Right Ventricular Function } & RV End Diastolic Volume $\left(\mathrm{mL} \cdot \mathrm{m}^{2}\right)$ & 79 & $66-126$ \\
\hline & RV End Systolic Volume $\left(\mathrm{mL} \cdot \mathrm{m}^{2}\right)$ & 35 & $19-59$ \\
\hline & RV Stroke Volume (mL) & 43 & $41-73$ \\
\hline & RV Ejection Fraction (\%) & 54 & $41-71$ \\
\hline Strain Imaging & Average Lateral Ventricular Systolic Strain (\%) & $14^{*}$ & $>18$ \\
\hline Ventricular Septum & Thickness (diastole) (mm) & $12 *$ & $8-11$ \\
\hline Posterior Wall & Thickness (diastole) (mm) & $16^{*}$ & $8-12$ \\
\hline Left Atrium & Volume Index by A-L Method $\left(\mathrm{cc} \cdot \mathrm{m}^{2}\right)$ & $33 *$ & $16-28$ \\
\hline Mitral Valve Diastolic & Deceleration Time (msec) & $233^{*}$ & $144-220$ \\
\hline Pulmonary Valve Systolic (Valve Area) & PV Peak Velocity (CWD) $\left(\mathrm{m} \cdot \mathrm{sec}^{-1}\right)$ & $1.2 *$ & $0.4-1.0$ \\
\hline MRI of the Heart & Apical Septal Wall Thickness (mm) & $24 *$ & $5-15$ \\
\hline
\end{tabular}

Asterick $(*)$ indicates the value is different from the normal range.

Briefly, standard echocardiographic data included left ventricular (LV) size and systolic function, including resting left ventricular outflow tract (LVOT) velocity, and peak systolic strain were examined. Ejection fraction was determined using either visual estimate, 2D (Quinone's method) or M-mode echocardiography. Maximal wall thickness was assessed using the $2 \mathrm{D}$ parasternal view to obtain measurements from the thickest portion of the ventricular septum. In all cases, the septum was the thickest portion of the left ventricular myocardium. Left atrial (LA) size was determined by measuring the $2 \mathrm{D}$ area of the left atrium obtained in the standard four-chamber and twochamber apical views in both the long- and short-axis and averaging the results. Other LV diastolic function parameters, including $\mathrm{E}$ peak velocity, $\mathrm{e}^{\prime}$, medial mitral annulus velocity, tricuspid regurgitation velocity, and pulmonary vein flow velocities were also evaluated using standard echocardiography procedures [24, 39].

\section{Magnetic Resonance Imaging (MRI)}

Cardiac MRI was used to localize, quantify and diagnose the apical HCM. Magnetic resonance imaging is of particular value in HCM when used together with two-dimensional echocardiography to document the site and extent of hypertrophy, especially in apical HCM [40].
All images were scanned on a 1.5 Tesla GE Sigma Excite Twin Speed clinical scanner (General Electric Medical Systems, Milwaukee, WI, USA) using appropriate 4- or 8element cardiac phased-array receiver coil. The cardiac MRI scans were taken under normal resting conditions at approximately $0800 \mathrm{hr}$ following a good night's sleep. Multiple short- and long-axis slices were recorded from the base to the apex of the entire ventricles. The cardiac MRI scans provided here (Fig. 4) are used to illustrate and confirm apical HCM.

\section{Statistical Analyses}

The HCM subject and group data are expressed as mean $( \pm \mathrm{SD})$ where applicable. The comparison of cardiac performance dependent variables taken from the BCG (timing and amplitude) between the HCM subject and group data was conducted using an independent Student T-test, with significance set at alpha $\mathrm{p} \leq 0.05$.

\section{RESULTS}

\section{Physical Characteristics}

The physical characteristics for all athletes reveal that the group was relatively homogeneous, with the HCM subject having less body fat $(9.7 \%$ vs $7.2 \%)$. The physiological fitness testing results for all athletes are presented in Table 1a (anaerobic and aerobic fitness), and $1 \mathrm{~b}$ and $1 \mathrm{c}$ (musculoskeletal fitness). These results showed that the 
HCM subject had a similar anaerobic, aerobic and musculoskeletal fitness as the cohort group of hockey players, indicating a high level of physical fitness.

\section{Electrocardiogram (ECG)}

The ECG showed waveform patterns typical of HCM. Although the HCM subject was in normal sinus rhythm throughout the medical assessment (range $=52-123$ beats $/$ minute; average $=67$ beats $/$ minute), Fig. (1) shows deeply inverted T-waves in the precordial leads, and resting ST segment abnormality considered to be anterolateral ischaemia. No premature ventricular complexes (PVC's) were evident, only occasional supraventricular premature complexes (SVPC) were observed. The PR interval and QRS duration were normal at $164 \mathrm{msec}$ and $102 \mathrm{msec}$, respectively.

During the maximal incremental cycle test at the medical and fitness assessment combine (and the advanced cardiac stress assessment laboratory), the HCM subject reached maximal levels of exertion $\left(\mathrm{VO}_{2} \max =59.4 \mathrm{~mL} \cdot \mathrm{kg}^{-1} \cdot \mathrm{min}^{-1}\right.$; $200 \mathrm{~b} \cdot \mathrm{min}^{-1}$ ) and the test was terminated when there was no further increase in $\mathrm{VO}_{2}$ with increasing work levels. The maximal stress test ECG was abnormal, but there was a normal blood pressure and heart rate frequency response to maximal exercise. No chest pain was reported during the stress test and only minor arrhythmias were noted.

\section{Ballistocardiogram (BCG)}

The BCG waveforms are presented in Fig. (2a-e), and the calculated cardiac timing and amplitude changes from the BCG are presented in Fig. (3a, b). These data demonstrate both normal and abnormal cardiac cycle performance.

\section{Cardiac MRI}

The final impressions of the cardiac MRI for the HCM subject confirm apical morphological subtype hypertrophic cardiomyopathy with the greatest thickness measuring 24-25 $\mathrm{mm}$ in the apical septum (Fig. 4). Closer evaluation showed asymmetrical hypertrophy of the left ventricle with patchy foci of delayed myocardial enhancement within the hypertrophied portion of the apex consistent with fibrosis. The anterior septal segments at the base measured 12-13 $\mathrm{mm}$. Systolic function was preserved, with no dynamic midcavity or LV outflow tract (LVOT) obstruction, and systolic anterior motion of the mitral valve (SAM) was not detected. Normal cardiac MRI values are reported in Table $\mathbf{2}$ for comparison.

\section{Echocardiography (Echo)}

The Echo results for the pathological HCM subject are illustrated in Table 2, and showed that clinical differences exist between the HCM subject and normal valves reported in the literature [24, 41]. Fig. (5) shows an apical 2D view confirming \#1 apical HCM (New York Heart Association Class 1) in this subject.

\section{DISCUSSION}

Hypertrophic cardiomyopathy is the leading cause of unexpected sudden cardiac death in young athletes [2, 42, 43]. Echocardiography and cardiac MRI are non-invasive techniques that are most specific and sensitive to successfully diagnose athletes with HCM [44-46]. However, these techniques are not always available and are expensive to perform, especially for mass testing of athletic teams. Thus, a simple and effective screening method that would reduce the cost of testing and identify cardiac anomalies would be appropriate initially. We believe our study is novel in two respects: (1) in a review of the literature we found no studies that used BCG for screening elite athletes [47, 48], and (2) the BCG did detect a cardiac abnormality in a subject at rest which was later diagnosed and confirmed by a cardiac consultant to be \#1 Apical HCM, New York Heart Association Class 1 by using the combined findings from the ECG, Echo and cardiac MRI [49].

Some researchers have suggested that testing every athlete is impractical [7]. However, we believe that BCG has the potential to be used as a front-line screening device during mass testing of athletes. Furthermore, based on the novel results of our study we suggest that combined ECG and BCG could potentially be used as an inexpensive screening procedure for mass athletic testing to assess cardiac abnormalities [44]. Compromised athletes could then be sent for advanced medical follow-up (Echo, cardiac MRI) as conducted here in an attempt to differentiate the "athletic" heart from a pathological condition [24, 46].

During mass athletic testing, one goal would be to detect the athlete that is predisposed to an abnormal electrical or mechanical functioning of the heart $[2,50]$. Together with ECG, our efforts using BCG in this study allowed us to detect an abnormal mechanical functioning of the heart under resting conditions. Although Echo is more sensitive and specific for HCM identification (see Figs. 4, 5), the advantage of adding BCG to the screening protocol is that cardiac timing and waveform amplitude $(\mathrm{mG})$ can be determined and compared with the entire athletic group to differentiate suspected athletes based on mechanical cardiac variables, and this can be performed in a much shorter time period (3-4 minutes for BCG vs 20-30 minutes for Echo). Furthermore, BCG has been correlated with Echo previously [34].

As shown in Figs. (2a-e), we noted structurally abnormal waveforms in both the diastolic and systolic phases of the cardiac cycle (Fig. 2c-e) in comparison to a representative player (Fig. 2a). Previous research has shown that individuals diagnosed with HCM demonstrated diastolic dysfunction [5, 21], and it is suggested that this can be differentiated from those individuals that have physiological hypertrophy resulting from exercise training [21, 44, 51, 52]. In Fig. (2c), it can be seen immediately following the early diastolic (ED) filling wave (Ewave on Echo) an unusually "aberrant" waveform appeared which reflects what we think is regurgitation following mitral valve open (MVO). Likewise, as noted in Fig. (2e) we document a bifurcation on the MVO signal. During the followup comprehensive assessment, the attending cardiac consultant noted mild mitral valve regurgitation from the Echo images. Based on the Echo data presented in Table 2, this subject has a diastolic function abnormality (e.g., deceleration time, 233 msec). The BCG data also demonstrated that this HCM subject had shorter diastolic phase when compared to the group cohort $(470.8 \pm 25.3$ vs $531.4 \pm 167.7 \mathrm{msec} ; \mathrm{p}<0.05)$. A reduced diastolic filling period could suggest a "stiffer" heart and/or reflect differences in flow dynamics, which was evident from the late diastolic filling (LD) wave amplitude (Fig. 3b). 

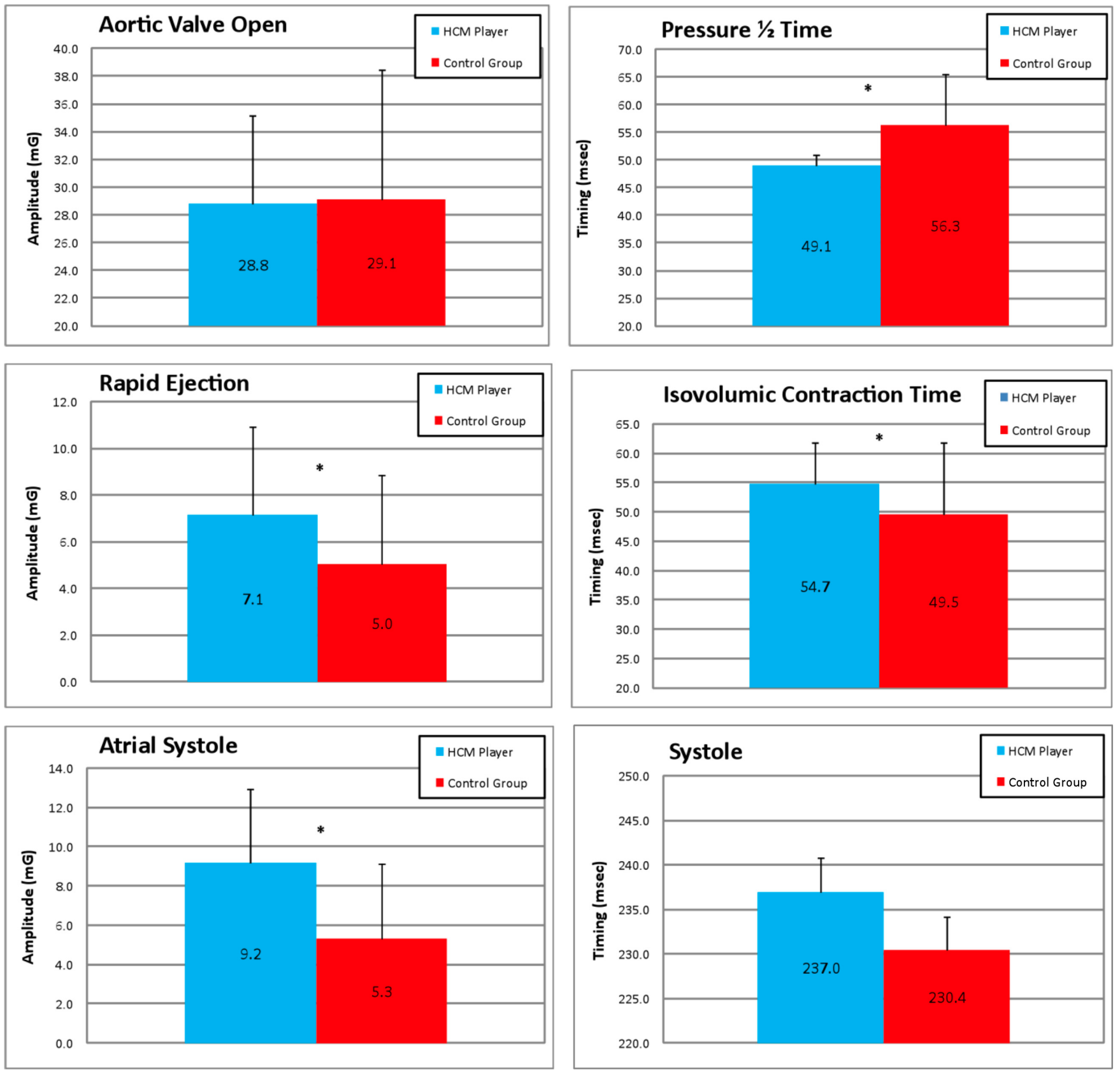

Fig. (3a). Systolic timing (msec) and amplitude $(\mathrm{mG})$ results for the HCM subject in comparison to cohort group (n=33) means taken from the BCG. Values are mean \pm SD based on $31 \pm 6$ annotated waveforms of the BCG. Asterisks $\left(^{*}\right)$ indicates significantly different between the HCM subject and group mean $(\mathrm{p} \leq 0.05)$.

The BCG waveform shown in Fig. (2d) presents a bifurcation on the aortic valve open (AVO). However, upon further examination of the Echo results we determined that this may be latency in the opening of the pulmonic valve, with the first peak confirming the AVO, and the second peak resembling the pulmonic valve opening signal (personal communication, Dr. Kwan-Leung Chan, Ottawa Heart Institute). The latency period between the opening of the aortic valve and pulmonic valve for the HCM subject was on average $12.6 \pm 1.6 \mathrm{msec}$, with $77 \%$ of his systolic AVO waveforms exhibiting this split. We noted that in the BCG (Fig. 2a) of a representative hockey player randomly selected from the group the frequency of this bifurcation was $<1 \%$. However, of all other players $(\mathrm{n}=33)$ tested during the professional assessment combine, $\sim 29 \%(\mathrm{n}=9)$ showed this aberrant waveform. Future research will help to elucidate the mechanism of this event, or whether it is pathological or a physiological adaptation to the high intermittent exercise training performed in ice hockey. Current heart failure literature suggests that this observation is reflective of interventricular dyssynchrony [53].

We were interested in comparing both the timing and amplitude (contractility) changes between the total group and the HCM subject. Although many of the timing parameters taken from the BCG revealed significant differences between 

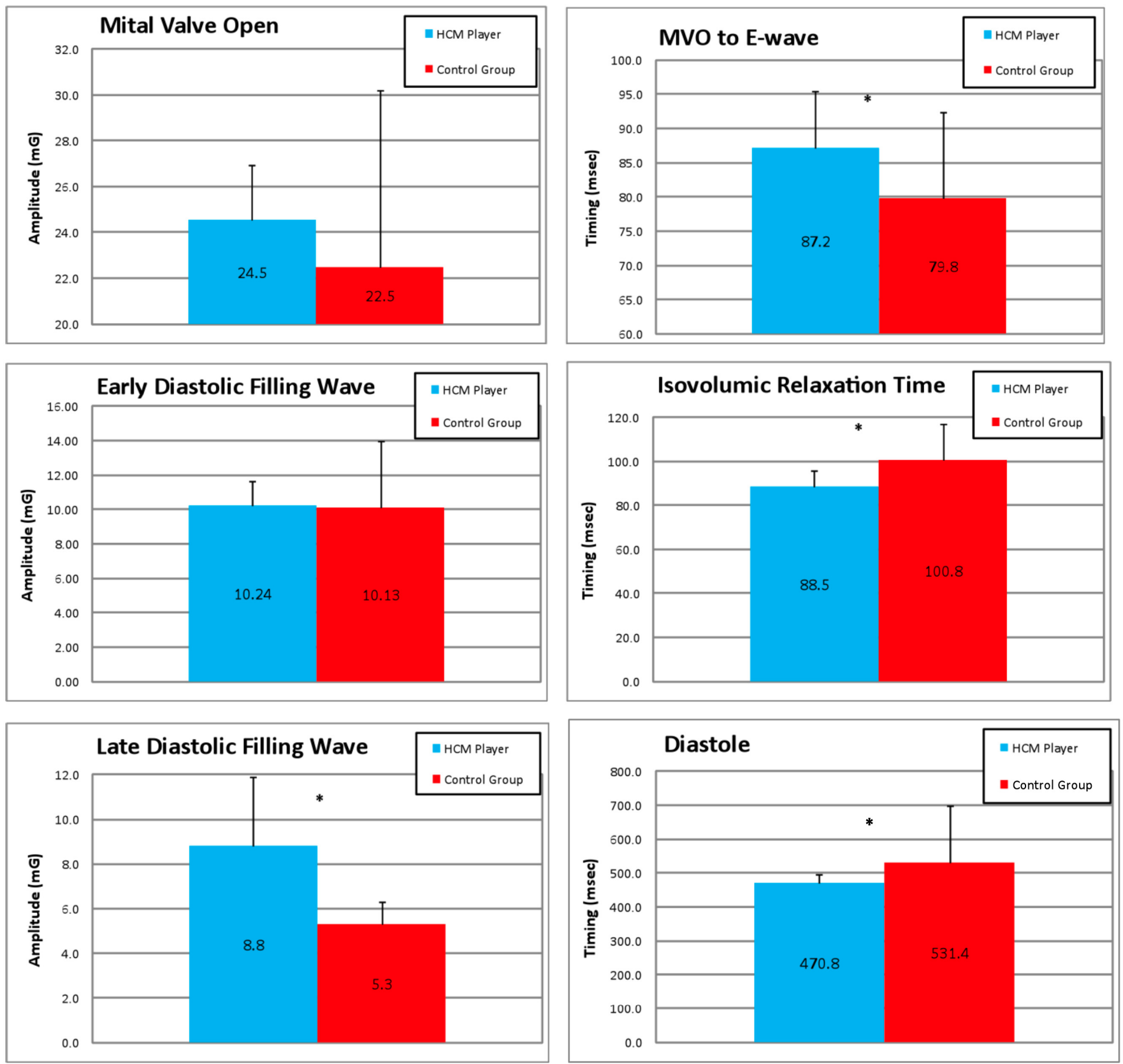

Fig. (3b). Diastolic timing (msec) and amplitude $(\mathrm{mG})$ results for the HCM subject in comparison to cohort group ( $\mathrm{n}=33$ ) means taken from the BCG. Values are mean \pm SD based on $31 \pm 6$ annotated waveforms of the BCG. Asterisks $\left(^{*}\right)$ indicates significantly different between the HCM subject and group mean $(\mathrm{p} \leq 0.05)$.

the HCM subject and the group mean, we selected representative cardiac cycle systolic (Fig. 3a) and diastolic (Fig. 3b) events for ease of comparison. From this data, we observed that during systole the pressure half-time $\left(\mathrm{P}^{1 / 2} \mathrm{~T}\right.$; time between AVO and rapid ejection) was significantly shorter $(49.1 \pm 1.8$ vs $56.3 \pm 9.1 \mathrm{msec})$, and isovolumic contraction time (IVCT) was significantly longer $(54.7 \pm 7.1$ vs $49.5 \pm 12.4 \mathrm{msec}$ ) in our HCM subject. In addition, cardiac contractility as defined here by the amplitude of the waveforms, showed atrial systole $(9.2 \pm 3.7$ vs $5.3 \pm 3.0 \mathrm{mG})$ and rapid ejection $(7.1 \pm 3.8$ vs $5.0 \pm 3.8 \mathrm{mG})$ were significantly higher in the HCM subject. This observation would indicate that transmitral pressure was elevated in the HCM subject, which was confirmed by the Echo data. Thus, collectively the BCG systolic data would suggest a more powerful systolic ejection (likely related to LV and septal mass) and an elevated atrial pressure (as indicated by the increased atrial systolic filling wave) in the HCM subject.

During diastole, the isovolumic relaxation time (IVRT) $(88.5 \pm 7.4$ vs $100.8 \pm 16.0 \mathrm{msec})$ and the diastolic filling period $(470.8 \pm 25.3$ vs $531.4 \pm 167.7 \mathrm{msec})$ were significantly shorter for the HCM subject and only the late diastolic filling wave (LD) was significantly higher in amplitude $(8.8 \pm 3.1$ vs $5.3 \pm 3.0 \mathrm{mG})$ in the HCM subject (Fig. 3b). Taken together, these results suggest that the HCM subject has altered diastolic function relative to his cohorts. The Echo data support the BCG observations. Lauschke and 

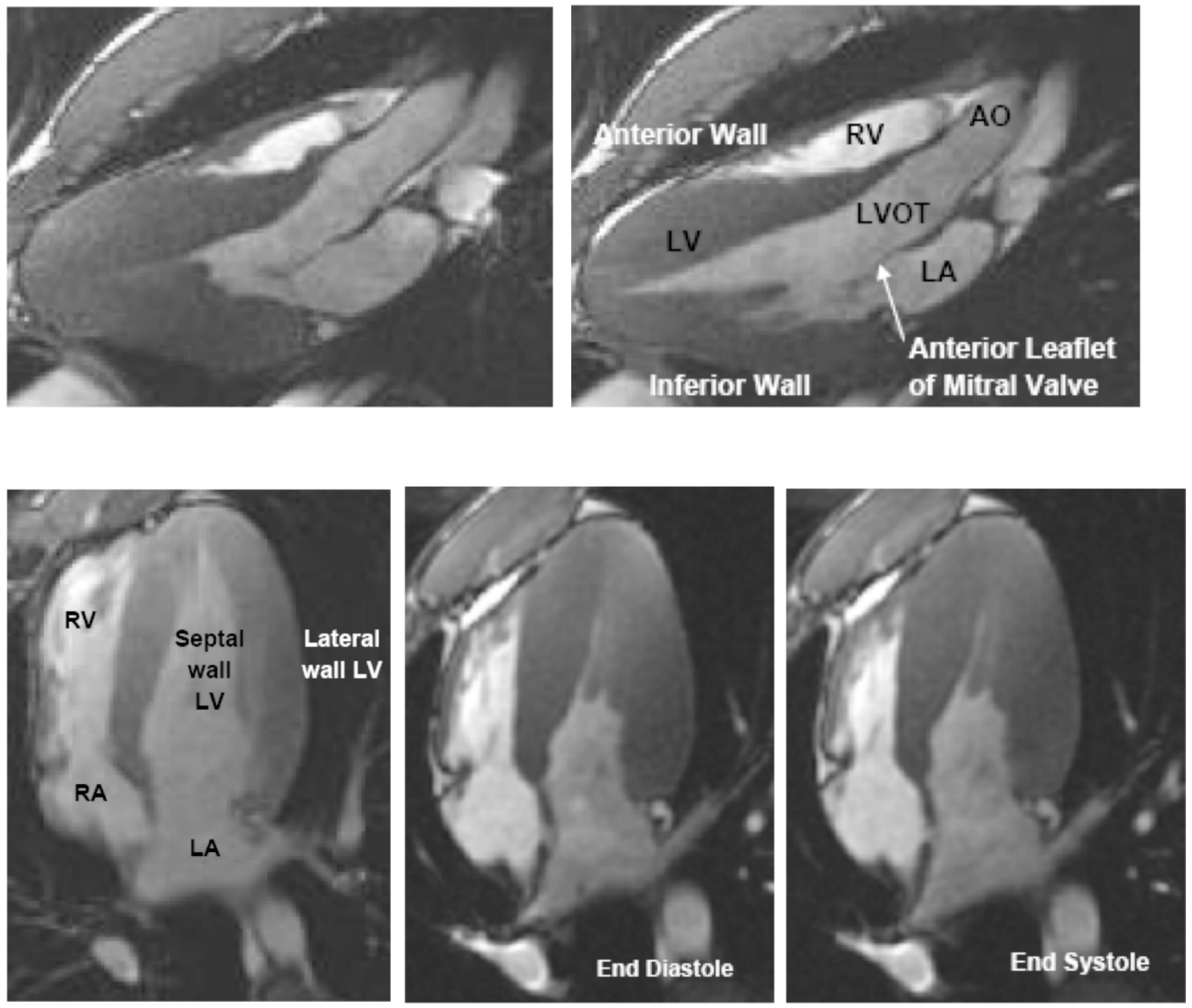

Fig. (4). Cardiac magnetic resonance imaging (MRI) images of the hypertrophic cardiomyopathy (HCM) hockey player's heart illustrating apical hypertrophy of the ventricular cardiac muscle mass during both systole and diastole.

co-workers have suggested that changes in diastolic function maybe important to help clarify differences between the "athletic" and "pathological" heart [54], and thus warrants further investigation.

This study advances our understanding of HCM, but more detailed studies comparing Echo, cardiac MRI and BCG are required to confirm the sensitivity and specificity of the BCG in detecting cardiac anomalies. In its present form, the BCG sensor is not specific to different clinical cardiac conditions. However, the BCG sensor used in this study was extremely sensitive to cardiac performance changes and wall motion. This was clearly illustrated from the BCG tracings displayed in Fig. (2a-e). With this device we also analyzed on average $31 \pm 6$ BCG waveforms for all players $(n=34)$ relatively quickly, and thus generated a large data set so that we could examine any number of cardiac performance variables. In its present state, only an "abnormal" BCG waveform can be identified but this identification lead us to probe further and perform a more thorough examination of the ECG, resulting in a more comprehensive cardiac assessment of this elite ice hockey player. Furthermore, the added value of the BCG is that cardiac cycle timing and durations can be quickly calculated and can then be compared directly with group results to determine potential "outliers". However, another limitation of BCG is that it cannot be used to differentiate between non-pathological and pathological HCM.

\section{CONCLUSION}

Although basic ECG continues to be a vital and cost effective front-line screening tool [44], it is our contention that the simultaneous measurement of BCG can provide additional mechanical information to confirm cardiac abnormalities in the mass screening of athletes when Echo and cardiac MRI are precluded. For example, BCG can provide timing, duration and amplitude changes of the cardiac cycle waveforms reflecting mechanical function (and dysfunction) and these can then be compared to normative data established by Echo. Our results support the use of non- 
invasive BCG for the mass screening of young athletes, which may help to confirm "at-risk" individuals. Further research is needed on other groups of athletes to help ascertain the utility of BCG as a screening tool.
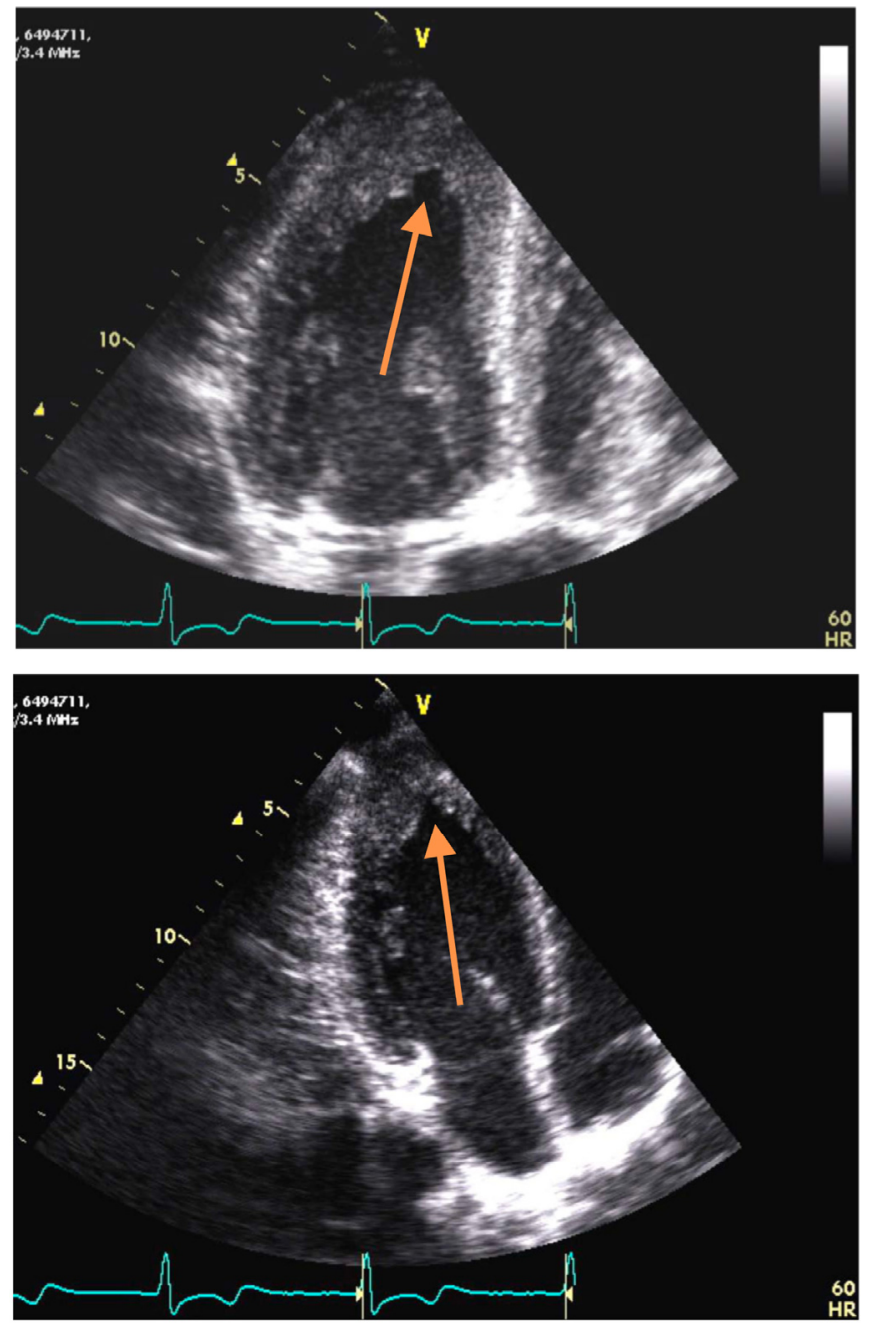

Fig. (5). Two dimensional echocardiography images showing the apical view of the localized hypertrophy in the left ventricular apex at end diastole. Note the spade-like configuration of the apex. The arrow indicates the direction of the apex.

\section{ACKNOWLEDGEMENTS}

We wish to thank our subjects for allowing us to share this critical research data with the scientific community, and to Dr. Steve R. Ommen of the Mayo Clinic in Rochester, MN, USA, for providing the Echo and cardiac MRI images and his editorial comments in the preparation of our manuscript. Thanks to Dr. Vern Gebhardt and Dr. Ron Schulte for their assistance with the Echo and cardiac MRI interpretation. We are in debt to the NHL Central Scouting Combine for allowing us to collect this data on subjects involved in the Combine. Research funding was provided by the Canadian Institutes of Health Research (JPN), Saskatchewan Health Research Foundation (JPN), and Heart Force Medical Inc.

\section{DISCLOSURE}

Dr. Neary has received Shares in Heart Force Medical Inc. for his research contribution toward the BCG device.
Mr. D. MacQuarrie is a graduate student and co-inventor of the BCG device and Dr. E. Busse has Shares in Heart Force Medical, and therefore could potentially benefit financially from the selling of this device. Drs. Jamnik, N. Gledhill, and S. Gledhill have no disclosures. Dr. Neary and D. MacQuarrie no longer consult to HFM Inc.

\section{REFERENCES}

[1] Cardim N, Oliveira AG, Longo S, et al. Doppler tissue imaging: Regional myocardial function in hypertrophic cardiomyopathy and in athlete's heart. J Am Soc Echocardiogr 2003; 16(3): 223-32.

[2] Rowland T. Sudden unexpected death in young athletes: reconsidering "hypertrophic cardiomyopathy". Pediatrics 2009; 123(4): 1217-22.

[3] Popovic D, Popovic N, Stojiljkovic S, Nesic D, Scepanovic L. [Distinguishing the athlete's heart syndrome from some pathological conditions]. Med Pregl 2008; 61(9-10): 483-8.

[4] Maron BJ, Gardin JM, Flack JM, Gidding SS, Kurosaki TT, Bild DE. Prevalence of hypertrophic cardiomyopathy in a general population of young adults. Echocardiographic analysis of 4111 subjects in the CARDIA Study. Coronary Artery Risk Development in (Young) Adults. Circulation 1995; 92(4): 785-9.

[5] Soor GS, Luk A, Ahn E, et al. Hypertrophic cardiomyopathy: current understanding and treatment objectives. J Clin Pathol 2009; 62(3): 226-35.

[6] Maron BJ. Ventricular arrhythmias, sudden death, and prevention in patients with hypertrophic cardiomyopathy. Curr Cardiol Rep 2000; 2(6): 522-8.

[7] Firoozi S, Sharma S, McKenna WJ. Risk of competitive sport in young athletes with heart disease. Heart 2003; 89(7): 710-4.

[8] Friedewald VE, Maron BJ, Roberts WC. The editor's roundtable: sudden cardiac death in athletes. Am J Cardiol 2007; 100(9): 14519.

[9] Kohl HW 3rd, Powell KE, Gordon NF, Blair SN, Paffenbarger RS Jr. Physical activity, physical fitness, and sudden cardiac death Epidemiol Rev 1992; 14: 37-58.

[10] Corrado D, Basso C, Schiavon M, Thiene G. Screening for hypertrophic cardiomyopathy in young athletes. N Engl J Med 1998; 339(6): 364-9.

[11] Papadakis M, Sharma S. Electrocardiographic screening in athletes: the time is now for universal screening. Br J Sports Med 2009; 43(9): 663-8

[12] Papadakis M, Chandra N, Sharma S. Controversies relating to preparticipation cardiovascular screening in young athletes: time for a realistic solution? Br J Sports Med 2011; 45(3): 165-6.

[13] Wyman RA, Chiu RY, Rahko PS. The 5-minute screening echocardiogram for athletes. J Am Soc Echocardiogr 2008; 21(7): 786-8.

[14] Basavarajaiah S, Wilson M, Whyte G, Shah A, McKenna W, Sharma S. Prevalence of hypertrophic cardiomyopathy in highly trained athletes: relevance to pre-participation screening. J Am Coll Cardiol 2008; 51(10): 1033-9.

[15] Pelliccia A, Di Paolo FM, Corrado D, et al. Evidence for efficacy of the Italian national pre-participation screening programme for identification of hypertrophic cardiomyopathy in competitive athletes. Eur Heart J 2006; 27(18): 2196-200.

[16] Corrado D, Basso C, Schiavon M, Pelliccia A, Thiene G. Preparticipation screening of young competitive athletes for prevention of sudden cardiac death. J Am Coll Cardiol 2008; 52(24): 1981-9.

[17] Ommen SR, Nishimura RA. Hypertrophic cardiomyopathy. Curr Probl Cardiol 2004; 29(5): 239-91.

[18] Ommen SR, Shah PM, Tajik AJ. Left ventricular outflow tract obstruction in hypertrophic cardiomyopathy: past, present and future. Heart 2008; 94(10): 1276-81

[19] Maron BJ. Distinguishing hypertrophic cardiomyopathy from athlete's heart physiological remodelling: clinical significance, diagnostic strategies and implications for preparticipation screening. Br J Sports Med 2009; 43(9): 649-56.

[20] Whyte GP, George K, Sharma S, et al. The upper limit of physiological cardiac hypertrophy in elite male and female athletes: the British experience. Eur J Appl Physiol 2004; 92(4-5): 592-7.

[21] Lauschke Jr, Maisch B. Athlete's heart or hypertrophic cardiomyopathy? 2009; 98(2): 80-8. 
[22] Scharhag J, Schneider G, Urhausen A, Rochette V, Kramann B, Kindermann W. Athlete's heart: right and left ventricular mass and function in male endurance athletes and untrained individuals determined by magnetic resonance imaging. $\mathrm{J}$ Am Coll Cardiol 2002; 40(10): 1856-63.

[23] Hansen MW, Merchant N. MRI of hypertrophic cardiomyopathy: part I, MRI appearances. AJR Am J Roentgenol 2007; 189(6): 1335-43.

[24] Kansal MM, Lester SJ, Surapaneni P, et al. Usefulness of twodimensional and speckle tracking echocardiography in "gray zone" left ventricular hypertrophy to differentiate professional football player's heart from hypertrophic cardiomyopathy. Am J Cardiol 2011; 108(9): 1322-6.

[25] Scarborough WR, Baker BM, Davis FW Jr., Mason RE, Singewald ML, Sherwin RW. Ballistocardiography and coronary heart disease: Prognostic evaluation based on a long-term follow-up study. Bibl Cardiol 1968; 20: 57-81.

[26] Scarborough WR, Davis FW Jr, Baker BM Jr, et al. A ballistocardiographic study of 369 apparently normal persons: An analysis of normal and borderline ballistocardiograms. Am Heart J 1953; 45(2): 161-89.

[27] Starr I. The place of the ballistocardiogram in a Newtonian cardiology and the new light it sheds on certain old clinical problems. Proc R Soc Med 1967; 60(12): 1297-306.

[28] Starr I. Beat-to-beat and minute-to-minute variation of cardiac function in abnormal hearts. Bibl Cardiol 1973; 0(31): 260-3.

[29] Salerno DM, Zanetti J. Seismocardiography for monitoring changes in left ventricular function during ischemia. Chest 1991; 100(4): 991-3.

[30] Salerno DM, Zanetti JM, Green LA, Mooney MR, Madison JD, Van Tassel RA. Seismocardiographic changes associated with obstruction of coronary blood flow during balloon angioplasty. Am J Cardiol 1991; 68(2): 201-7.

[31] Carlsson J, Erdogan A, Rolf A, et al. [Recurrent syncope in a 34year-old woman triathlete]. Dtsch Med Wochenschr 2000; 125(37): 1074-8.

[32] Pelliccia A, Maron BJ, Culasso F, et al. Clinical significance of abnormal electrocardiographic patterns in trained athletes. Circulation 2000; 102(3): 278-84.

[33] Basso C, Maron BJ, Corrado D, Thiene G. Clinical profile of congenital coronary artery anomalies with origin from the wrong aortic sinus leading to sudden death in young competitive athletes. J Am Coll Cardiol 2000; 35(6): 1493-501.

[34] Crow RS, Hannan P, Jacobs D, Hedquist L, Salerno DM. Relationship between seismocardiogram and echocardiogram for events in the cardiac cycle. Am J Noninvas Cardiol 1994; 8: 39-46.

[35] Afonso LC, Bernal J, Bax JJ, Abraham TP. Echocardiography in Hypertrophic Cardiomyopathy: The Role of Conventional and Emerging Technologies. JACC: Cardiovasc Imaging 2008; 1(6): 787-800.

[36] Scarborough WR, Talbot SA. Proposals for ballistocardiographic nomenclature and conventions: Revised and extended report of committee on ballistocardiographic terminology. Circulation 1956; 14(3): 435-50

[37] Burr JF, Jamnik RK, Baker J, Macpherson A, Gledhill N, McGuire EJ. Relationship of physical fitness test results and hockey playing potential in elite-level ice hockey players. J Strength Cond Res 2008; 22(5): 1535-43.
[38] Binder J, Ommen SR, Gersh BJ, et al. Echocardiography-guided genetic testing in hypertrophic cardiomyopathy: septal morphological features predict the presence of myofilament mutations. Mayo Clin Proc 2006; 81(4): 459-67.

[39] Blauwet LA, Ackerman MJ, Edwards WD, Riehle DL, Ommen SR. Myocardial fibrosis in patients with symptomatic obstructive hypertrophic cardiomyopathy: correlation with echocardiographic measurements, sarcomeric genotypes, and pro-left ventricular hypertrophy polymorphisms involving the renin-angiotensinaldosterone system. Cardiovasc Pathol 2009; 18(5): 262-8.

[40] Gaudio C, Pelliccia F, Tanzilli G, Mazzarotto P, Cianfrocca C, Marino B. Magnetic resonance imaging for assessment of apical hypertrophy in hypertrophic cardiomyopathy. Clin Cardiol 1992; 15(3): 164-8.

[41] Olivotto I, Maron MS, Autore C, et al. Assessment and Significance of Left Ventricular Mass by Cardiovascular Magnetic Resonance in Hypertrophic Cardiomyopathy. J Am Coll Cardiol 2008; 52(7): 559-66

[42] Corrado D, Basso C, Schiavon M, Thiene G. Screening for Hypertrophic Cardiomyopathy in Young Athletes. N Engl J Med 1998; 339(6): 364-9.

[43] Maron BJ, Doerer JJ, Haas TS, Tierney DM, Mueller FO. Sudden deaths in young competitive athletes: analysis of 1866 deaths in the United States, 1980-2006. Circulation 2009; 119(8): 1085-92.

[44] Corrado D, Biffi A, Basso C, Pelliccia A, Thiene G. 12-lead ECG in the athlete: Physiological vs pathological abnormalities. Br J Sports Med 2009; 43(9): 669-76.

[45] Teske AJ, Prakken NH, De Boeck BW, Velthuis BK, Doevendans PA, Cramer MJ. Echocardiographic deformation imaging reveals preserved regional systolic function in endurance athletes with left ventricular hypertrophy. Br J Sports Med 2010; 44(12): 872-8.

[46] Prakken NH, Velthuis BK, Cramer MJ, Mosterd A. Advances in cardiac imaging: the role of magnetic resonance imaging and computed tomography in identifying athletes at risk. Br J Sports Med 2009; 43(9): 677-84.

[47] Elsbach H, Rodrigo FA, Verbiest E. Further observations on the ballistocardiogram in hypertrophic cardiomyopathy. Bibl Cardiol 1968; $21: 125-9$.

[48] Phibbs B, Lowe CR, Holmes RW. The ultra low frequency force ballistocardiograph in acute cardiomyopathy. Circulation 1967; 36(1): 92-100.

[49] Hansen MW, Merchant N. MRI of Hypertrophic Cardiomyopathy: Part I, MRI Appearances. Am J Roentgenol 2007; 189(6): 1335-43.

[50] Link MS. Prevention of sudden cardiac death: Return to sport considerations in athletes with identified cardiovascular abnormalities. Br J Sports Med 2009; 43(9): 685-9.

[51] Lawless CE, Best TM. Electrocardiograms in athletes: interpretation and diagnostic accuracy. Med Sci Sports Exerc 2008; 40(5): 787-98.

[52] Lewis JF, Spirito P, Pelliccia A, Maron BJ. Usefulness of Doppler echocardiographic assessment of diastolic filling in distinguishing "athlete's heart" from hypertrophic cardiomyopathy. Br Heart J 1992; 68(3): 296-300.

[53] Bleeker GB, Bax JJ, Steendijk P, Schalij MJ, van der Wall EE. Left ventricular dyssynchrony in patients with heart failure: Pathophysiology, diagnosis and treatment. Nat Clin Pract Cardiovasc Med 2006; 3(4): 213-9.

[54] Lauschke J, Maisch B. Athlete's heart or hypertrophic cardiomyopathy? Clin Res Cardiol 2009; 98(2): 80-8. 